\title{
Developmental Regulation Before and After a Developmental Deadline: The Sample Case of "Biological Clock" for Childbearing
}

\author{
Jutta Heckhausen and Carsten Wrosch \\ Max Planck Institute for Human Development
}

\author{
William Fleeson \\ Wake Forest University
}

\begin{abstract}
This quasi-experimental research investigates developmental regulation around a critical life-span transition, the "biological clock" for childbearing. The action-phase model of developmental regulation proposes contrasting control orientations in individuals approaching versus those having passed a developmental deadline. Individuals in an urgency phase close to the deadline should be invested in goal pursuit, whereas those who have passed the deadline without attaining the goal should focus on goal disengagement and self-protection. In 2 studies, women at different ages and with or without children were compared with regard to various indicators of primary and secondary control striving for goal attainment versus goal disengagement and self-protection. Findings support the action-phase model of developmental regulation. Patterns of control striving congruent with the participants' status as preversus postdeadline were associated with superior psychological well-being.
\end{abstract}

The present line of research addresses individuals' attempts to regulate their own development when approaching and passing a developmental deadline for their developmental goals. Developmental deadlines represent final age-normative constraints for attaining developmental goals, which involve expected shifts from high to low opportunities (and low to high constraints) for achieving developmental goals (J. Heckhausen, 1999; Wrosch \& Heckhausen, 1999). Such expected shifts in goal attainability should bring about concomitant shifts in processes of developmental regulation from urgent goal striving to goal disengagement. Previous research in the domain of partnership has shown that individuals activate and deactivate developmental goals in accordance with age-graded changes in opportunities for goal attainment (Wrosch \& Heckhausen, 1999). In the two quasi-experimental studies presented here, we investigate processes of developmental regulation around a more discrete life-span transition, the deadline for childbearing associated with what is known as the "biological clock."

In the following discussion, we first introduce a general model of developmental regulation based on the life-span theory of control (J. Heckhausen \& Schulz, 1995; Schulz \& Heckhausen, 1996). Subsequently, we propose an action-phase model of devel-

Jutta Heckhausen and Carsten Wrosch, Center for Lifespan Psychology, Max Planck Institute for Human Development, Berlin, Germany; William Fleeson, Department of Psychology, Wake Forest University.

Jutta Heckhausen is now at the Department of Psychology and Social Behavior, School of Social Ecology, University of California, Irvine. Carsten Wrosch is now at the Department of Psychology, Concordia University, Montreal, Quebec, Canada.

We wish to thank Annette Rentz and Inga Schlesinger for their help with data collection and analyses.

Correspondence concerning this article should be addressed to Jutta Heckhausen, Department of Psychology and Social Behavior, School of Social Ecology, 3340 Social Ecology II, University of California, Irvine, California92697-7085. Electronic mail may be sent to heckhaus@uci.edu. opmental regulation around developmental deadlines. Finally, we develop a set of hypotheses about the adaptivity of processes individuals may use when approaching and passing the developmental deadline of childbearing.

\section{A Model of Developmental Regulation}

On the basis of Rothbaum, Weisz, and Snyder's (1982) distinction of primary and secondary control, J. Heckhausen and Schulz (1995; Schulz \& Heckhausen, 1996) have proposed the life-span theory of control. Primary control striving refers to individuals' attempts to change the external world so that it fits the needs and desires of the individual. Secondary control striving, by contrast, addresses individuals' efforts to influence their own motivation, emotion, and mental representations.

Developmental regulation comprises two basic components (J. Heckhausen, 1999). First, individuals try to actively influence their own development by selecting personal goals (e.g., starting a career, finding a partner, having a child) to strive for. Second, individuals try to adapt to and compensate for failure experiences and to adjust to the constraints of a given developmental ecology (J. Heckhausen, 1997). Active striving for developmental goals requires the mobilization and selective investment of behavioral and motivational resources. This involves both primary (i.e., invest time and energy in goal striving) and secondary (i.e., enhancing motivational goal commitment) control strategies of selective investment (see detailed model in J. Heckhausen, 1999; J. Heckhausen \& Schulz, 1993). In particular, selective primary control processes refer to the investment of behavioral resources, such as time, effort, and skills into the pursuit of a chosen goal. In case internal resources prove to be insufficient, compensatory primary control processes may be activated in terms of recruiting other people's help or advice or using other external aids (e.g., hearing aid). Goal striving is supported motivationally by selective secondary control processes, which serve to focus motivational com- 




Figure 1. Action-phase model of developmental regulation. Note. Adapted from Developmental Regulation in Adulthood: Age-Normative and Sociostructural Constraints as Adaptive Challenges (p. 114), by J. Heckhausen, 1999, New York: Cambridge University Press. Copyright 1999 by Cambridge University Press. Adapted with permission of the publisher.

mitment to a chosen goal and shield it off from alternative goals and stimuli.

Adaptation to failure and aging-related constraints, however, require a different set of control strategies, which are referred to as compensatory secondary control processes. These compensatory secondary control strategies serve two objectives: (a) disengaging from the goal to which one was previously committed (e.g., devaluating the previous goal, enhancing alternative goals) and (b) protecting the self against negative evaluations resulting from a failure experience (e.g., self-protective causal attributions, selfenhancing social comparison with inferior others; see Wrosch \& Heckhausen, in press).

\section{Action-Phase Model of Developmental Regulation: Rubicon and Developmental Deadline}

The Rubicon model of action phases was developed in a nondevelopmental context to capture the essence of motivational processes in general action (H. Heckhausen, 1991; H. Heckhausen \& Gollwitzer, 1987). The critical claim of the Rubicon model of action phases is that motivational processes before and after the decision, that is, before and after transgressing the Rubicon, differ distinctly. Getting engaged with a chosen action goal is thus not a matter of continuously increasing motivational investment, but instead comprises a radical and discrete shift in motivational processing from predecisional weighing of alternatives to postdecisional preoccupation with the chosen option.

The action-phase model of developmental regulation (J. Heckhausen, 1999; see Figure 1) extends the Rubicon model by including another discrete and motivationally relevant transition in the model, the deadline. The deadline for action represents a point in time after which action opportunities in a particular domain are severely curtailed or eliminated (J. Heckhausen, 1999; Wrosch \& Heckhausen, in press). Thus, developmental deadlines are expected to be represented as final timing constraints for attaining personal goals in the development-related conceptions of individ- ual members of a given social community. Such timing constraints in goal attainability can be anticipated by the individual and thus set up phases of urgent goal striving just before the deadline is reached, and of disengagement after the deadline has been passed.

Figure 1 identifies on three levels (from top to bottom) the following aspects of the extended model of action phases: critical transition points, functions and challenges of the sequential action phases (predecisional, nonurgent and urgent predeadline, and postdeadline), and control strategies adaptive to meet these phasespecific challenges. First, the two critical transitions are the Rubicon, when intentions are formed, and the deadline, when the action ecology shifts to more constraints and fewer opportunities (see also J. Heckhausen, 1999; Wrosch \& Heckhausen, in press). The deadline marks a point in time,' after which action opportunities are no longer available or radically reduced.

Second, the extended model of action phases further differentiates the predeadline actional phase into a nonurgent and an urgent phase of goal-oriented primary control striving. Thus, if action is delayed for whatever reason, goal attainment has to come up against ever narrowing time constraints. To still attain the goal, the individual needs to invest enhanced effort and volitional commitment in this urgent predeadline phase. Thus, on the level of control processes (see third level from top of Figure 1), the urgency phase created by the deadline calls for increased and focussed behavioral (i.e., selective primary and compensatory primary control) and

\footnotetext{
' One could also extend the concept of developmental deadlines beyond the time dimension to situational action opportunities in general. Such a general concept might be conceived as a "transition to a condition of lost opportunities" and would include situations when, in the process of goal striving, the external or internal prerequisites for goal attainment are lost. Examples would be a teacher, who in his early career is confronted with radically vanishing job opportunities in the school system, or an athlete, who in the process of training for peak performance suffers an incapacitating and irreversible injury.
} 
motivational (i.e., selective secondary control) investment in goal striving. After passing the deadline, goal intentions have either been realized or failed. Those who failed the deadline (see postdeadline failure condition in Figure 1) need to shift radically from intense goal engagement to compensatory secondary control in terms of goal disengagement and self-protective interpretations. In this way, individuals experiencing deadline failures may protect their motivational resources for future primary control striving. In contrast, those who were successful (see postdeadline success condition in Figure 1) can invest in further primary control striving and capitalize on the action resources strengthened by their success, either in the same domain or in a different domain that might have been neglected during the deadline-related phase of life.

The first evidence for pre- and postdeadline control behavior comes from a study on striving to attain a partnership after separation in young and late midlife adults (Wrosch \& Heckhausen, 1999). Because of declining remarriage opportunities, late midlife adults as compared with young adults confront greatly deteriorated opportunities to find a new partner. After a separation adults in late midlife tend to disengage from partnership goals altogether, whereas younger adults step up their efforts to find a new partner. Moreover, the young and late midlife adults also exhibited actionphase congruent control strategies. Even more important, late midlife adults profited from goal disengagement in terms of improving their positive affect over a period of 15 months. Young adults, by contrast, suffered a deterioration of positive affect when they disengaged from partnership goals after a separation.

The present set of studies applies the deadline model to a more radical and discrete shift in opportunities for goal attainment, namely the biological clock for childbearing. Because of the discrete nature of the presumed shift in fertility around age 40 , childbearing is a prime example of an age-graded deadline that is firmly rooted in age-normative beliefs. For the majority of women it can be assumed that they do not have precise knowledge about their personal fertility potential at a given age in midlife. Instead, women base their personal expectations on common-sense notions about declining fertility around the end of the 3rd decade of life (Settersten \& Hagestad, 1996). The discrete nature of the childbearing deadline thus allows us to investigate hypotheses about contrasting motivational orientations in neighboring age groups, and therefore goes beyond previous comparisons of goal orientation in young and old adults (J. Heckhausen, 1997) or young and late midlife adults (e.g., Wrosch \& Heckhausen, 1999).

Developmental Regulation Around the Deadline for Childbearing: Research Rationale and General Hypotheses

The two quasi-experimental studies reported here use the biological clock phenomenon as a paradigm to study goal engagement and goal disengagement around developmental deadlines. Toward the end of the 3rd decade of life, commonly shared conceptions expect fertility in women to sharply decline. Therefore, the late $30 \mathrm{~s}$ may well be conceptualized as the developmental deadline for childbearing (Settersten \& Hagestad, 1996). Given that having a child is a common and desirable developmental goal and certainly part of the life agenda of developmental tasks (Havighurst, 1952), negotiating the deadline for childbearing with its implications of having versus never having a child should be a challenge of early midlife that most people would perceive as very engaging.
The general hypothesis of the two studies presented is that individuals use control strategies conducive to goal attainment when they approach developmental deadlines for important goals and that they use control strategies that promote disengagement and self-protection when they have passed developmental deadlines without attaining the goal. Specifically, individuals just prior to the deadline (i.e., the urgent group) will use intensified behavioral and motivational investments to strive for the goal of having a child. In contrast, individuals who just passed the deadline without having a child will use compensatory secondary control strategies to disengage from the goal and to protect self-esteem. Individuals who recently had a child will engage in further goal striving that builds on the success with the childbearing goal.

Finally, we go beyond descriptively investigating conceptually predicted differences between control behavior in the pre- and postdeadline groups by examining their associations with subjective well-being. Our prediction is that phase-congruent control processes will be associated with higher subjective well-being than phase-incongruent control processes. Specifically, control processes involved in goal striving should be selectively adaptive for predeadline women, whereas goal disengagement should be adaptive for postdeadline women. Conversely, goal engagement control behavior is expected to be detrimental for postdeadline women, whereas goal disengagement is expected to be maladaptive for predeadline women.

We chose a quasi-experimental (Cook \& Campbell, 1979) design for this research in spite of its disadvantages from an internal (as opposed to an external) validity perspective. By investigating groups composed of individuals who are members of these groups by virtue of age and life situation, we introduced a number of confounds. For example, our groups differed somewhat in biographical background, in motivation, and on other dimensions. Our approach was based on several considerations: First, we submitted that ecological (external) validity is an important, if not critical, aspect of developmental psychology. In fact, the life-span theory of control and its model of developmental regulation (optimization in primary and secondary control [OPS model]; see J. Heckhausen, 1999; J. Heckhausen \& Schulz, 1993; Schulz \& Heckhausen, 1996) were developed with the explicit intent to reflect the agegraded and multifaceted context of life-span development, so that age-graded contexts are conceptualized as inherent in motivational processes. The costs of a quasi-experimental approach were potential confounds. Methodological alternatives would have been to conduct a highly controlled experiment on deadlines involved in a laboratory task (e.g., time running out for the task) or a longitudinal study. Regarding the laboratory experiment, the disadvantage would have been the lack of knowledge about whether and to what extent differences between experimental groups are moderated or outweighed by contextual factors present in the developmental ecologies the participants live in. A longitudinal study would have been impossible to conduct because of the number of years the study would need to last, and because of the impossibly large sample we would have to start with in order to end up with sufficient numbers in each group.

On the basis of these considerations, we chose a quasiexperimental approach and included two variables in our assessment to estimate and statistically control the potential confound in our data. First, we asked participants of all groups about their wish for a child at any time during their life, with the intent to get a 
handle on systematic variations due to an individual's motivation to have a child irrespective of deadline. Second, for the incidental memory tasks we included baseline memory tests to control for interindividual and age-group-related differences in basic memory performance.

The two studies address engagement and disengagement with child-related goals and goals pertaining to other important domains of life. Study 1 extends this approach by assessing an indicator of cognitive processing, namely incidental memory for child-relevant sentences. Study 2 adds a direct assessment of goal-specific primary and secondary control strategies. In each of the two studies, pre- and postdeadline groups of women are compared, and moreover, the predictive value of phase-congruent (pre- vs. postdeadline) goal engagement, selective recall, and control strategies is investigated.

\section{Study 1}

To achieve an ecologically valid grouping of participants, we recruited women who fit into the three relevant phases (i.e., missed deadline, met deadline, and urgency) by criteria of their age and parental status. The missed deadline group comprised women aged between 40 and 46 years with no children; the met deadline group involved women between 19 and 44 years, whose first child was less than 1 year old; and the urgent group consisted of women between 27 and 33 years who had no child yet. The selection of age 40 as the deadline was based on surveys about general age-timing conceptions about family events (e.g., Settersten \& Hagestad, 1996). The range of ages within the urgency and missed deadline groups allows for testing potential age differences within these groups to see whether younger women experience urgency and disengagement, respectively, in the same way as somewhat older women in the respective group.

Two types of indicators of goal engagement and goal disengagement were used in this study. First, we asked participants to explicitly nominate their current personal goals. Second, we used an incidental recall task as a measure of the relative salience of goal-relevant versus goal-irrelevant information in the different participant groups. H. Heckhausen and colleagues demonstrated that individuals in the predecisional versus actional motivational phases differed in their thought contents, as measured by thought listing (H. Heckhausen \& Gollwitzer, 1987), and differed in the content of recalled information from incidental memory tasks (Gollwitzer, Heckhausen, \& Steller, 1990). We assessed incidental memory by presenting several experimenter-generated sentences on a computer screen from each of the 10 categories listed below. Later, participants were surprised by a memory test for those sentences.

We expected both types of indicators, goal nominations and information salience, to reflect the motivational mind set that is based on the participant's position relative to the deadline. Participants in an urgent predeadline mind set should nominate more goals directed at childbearing than participants who have passed the deadline or participants who have already attained the goal. Moreover, participants in the predeadline urgent mind set should be particularly receptive of goal-relevant information, especially when it is positive information that enhances goal commitment.

For the present study, we constructed 10 content categories of information relevant to childbearing based on the action-phase model of developmental regulation (J. Heckhausen, 1999). We expected four of these categories to be particularly salient to those just prior to the deadline (urgent), because we hypothesized that such individuals would prefer using those control strategies that promote motivational commitment to and behavioral investments in goal striving. Sentences about "means and opportunities" are relevant for selective primary control. The sentence categories "goal value good," "positive feedback," and "age timing" relate to major components of selective secondary control.

Four different categories were expected to be particularly salient to those women who passed the deadline without giving birth. We expected these passed deadline women to use compensatory secondary control strategies in support of disengaging from the goal and protecting one's motivational resources (e.g., self-esteem). Sentences in the categories "goal value bad," "competing goals," and "substitute goals" are relevant for goal disengagement, whereas the category "attributions that avoid responsibility" is related to the self-protection component of compensatory secondary control. We included one category expected to be salient to those women who attained the goal (i.e., had a child) because we expected them to engage in pursuing goals that capitalize on their success: "capitalizing goals." Finally, we expected the 10th category, "attributions that take responsibility," to be of depressed salience to individuals who failed to meet the deadline.

For the information salience task, all participants, regardless of group membership, were shown the same sentences. Furthermore, all sentences were written in non-self-referent language, participants were instructed to try to recall all sentences, and participants were surprised by the memory task. This methodology was meant to ensure that any group differences in the recall of sentences from different content categories are attributable to a group-differential salience of the content categories.

In addition to identifying patterns of sentence recall, which are adaptive for goal engagement and for goal disengagement, this study also investigates the implications of such patterns for psychological well-being. The adaptive value of patterns of information processing can be investigated from an interindividual difference perspective. Individuals may differ in the extent to which they are adapted to their respective motivational phase, thus, exhibiting different degrees of correspondence between salience of deadline and goal-relevant information and functional requirements of their current motivational phase. We therefore predict that, within a given motivational phase, those individuals who recall more sentences relevant to the functional requirements of their motivational phase should also report higher well-being.

\section{Method}

\section{Participants}

Our goal in sampling was to obtain a diverse set of individuals, who by age and parental status fall naturally into the conditions of interest in this study. Participants were recruited through newspaper and radio advertisements; posting announcements in universities, medical clinics, and fitness centers; and by contacting participants who had participated in previous unrelated (intelligence) studies conducted at the Max Planck Institute for Human Development.

Participants $(N=143)$ were invited to participate if they met the criteria for one of the three conditions: missed deadline if they were age 40 to 46 years ( $n=43, M=42.44$ years, $S D=1.69$ ) and had never had a child; 
met deadline if they were age 19 to 44 years $(n=49, M=29.83$ years, $S D=5.45$ ) and their only child was less than 1 year old; urgency if they were age 27 to 33 years $(n=51, M=29.80$ years, $S D=1.89)$ and had never had a child. Sixty-four percent $(n=92)$ of the participants were highly educated (German Abitur, which is equivalent to graduates of a college-preparatory high school, and higher). Participants received DM 40 (about \$23) for their participation.

\section{Procedure}

Participants completed the experiment in groups of 1 to 5 people. They sat at individual computers within the same room and completed materials on the computer and on questionnaires. The participants first completed a warm-up procedure, then participated in the incidental learning phase, completed an affect measure, participated in the recall phase, and finally completed a questionnaire. This questionnaire included a "retrospective childwish" question on whether they wanted a child (not at all, a little, somewhat, a lot, very much) at any 5-year period between 20 years of age and their current age. The entire experiment lasted between 1 and $2 \mathrm{hr}$, and participants who completed the experiment in the same group were conducted through most of the steps at the same rate.

Warm up procedure. First, participants completed a warm-up procedure to focus their attention on the issues of childbearing; this procedure was intended to reduce error variance due to distraction. Participants responded for $30 \mathrm{~min}$ total to the following four questions: "How do you feel, and what occurs to you, when you think about the fact that you have a child?" "How does it influence your life that you have a child?" "What are the most important reasons that you have a child?" "Was there a time in your life that you wished more or less than now for a child? Why?" For the participants with no children, the questions were identical except that "have a child" was substituted for "have no child."

Learning phase. After completing the warm-up procedure, participants began the learning phase of the experiment. Participants were told that they would see a series of sentences on the computer and that they were to press a number from 1 to 5 indicating their level of agreement with the sentence. They were told to respond as quickly as possible. Note that this study tests incidental memory: The experimenter gave no indication that the participants would be asked to recall the sentences later. After one practice sentence, each sentence appeared in the center of a blank screen until the participant pressed a number from 1 to 5 . If the participant pressed any other key, the sentence remained on the screen. If the participant pressed no number within $8 \mathrm{~s}$, the sentence disappeared from the screen and the next sentence appeared on the screen. Sentences appeared in a different random order for each participant.

Recall phase. After completing an affect measure, participants were surprised with a memory test. They were asked to recall as many of the sentences as possible and to recall them as close to word for word as they could. They were given 20 min to complete this task; the experimenter asked all participants to wait before continuing to the next task and after 20 min asked all participants to complete the remainder of the questionnaire.

\section{Materials}

Developmental goals. Participants' developmental goals were assessed with an open response format questionnaire (J. Heckhausen, 1997). Participants were requested to list five personal goals, plans, or wishes that they wanted to attain within the next 5 to 10 years. The answers were coded into 10 goal domains: childbearing goals, child-rearing goals, occupational goals, family goals, health goals, leisure goals, self-related goals, friendsrelated goals, financial goals, and societal goals. The reliability estimates obtained in previous studies for the categorization of goals into goal categories was $92 \%$ interrater agreement (J. Heckhausen, 1997).

Incidental memory. Sentences for the incidental memory measure were generated by the experimenters for each of the 10 categories listed above; 1 additional category of neutral (i.e., non-baby relevant) content was also developed in order to control for individual differences in general memory ability. For each content category, we created six sentences that in our view covered the category comprehensively and that were distinct from each other. The neutral category consisted of 10 sentences for the purpose of safeguarding the reliability of this measure. Sentences were selected to be of about equal length and difficulty across categories. However, we should be clear that we make no predictions about across-category main effects; our only interest is in group by category interactions, which are unlikely to be affected by differences between categories in sentence difficulty or length. Therefore, we believe that our efforts at matching across categories were sufficient for the present purposes.

Coding of the recall of the sentences was completed by a coder who was unaware of the hypotheses or of the condition of the participant. Each sentence written by a participant was coded both for the content of the sentence (i.e., which original sentence it matched) and for its degree of match to an original sentence presented in the learning phase of the experiment. A sentence was coded as a perfect match if it was identical to the original sentence except for a maximum of one or two words (and if any such minor word changes had no effect on the meaning of the sentence). A sentence was coded as a moderate match if it captured the main idea of one of the original sentences but differed in several words or in the order of the words. A sentence could be scored as a category match if it did not match any of the sentences, but it fit into one of the categories. Otherwise a sentence was scored as a nonmatch. A second coder coded a subset of the participants, and analyses showed high agreement between the two coders, both in degree of match and in content of match. The two coders agreed on 155 of 185 sentences $(84 \%)$. Only four disagreements concerned the category ( $98 \%$ agreement in categorization); the remainders were disagreements in degree of match.

Emotional well-being. To measure the individual's level of adaptivity, we included the Positive and Negative Affect Scale (PANAS; Watson, Clark, \& Tellegen, 1988) as measures of subjective well-being. The PANAS measured participants' level of affective well-being during the past year.

\section{Results}

To test our hypotheses, we address each of the first two sets of hypotheses in turn (that differential mind sets will be evident in reported developmental goals and incidental memory). We then address the third set of hypotheses, that individuals who remember well information that fits their motivational phase will also report higher levels of well-being. With regard to the variable "retrospective childwish," differences between the three quasi-experimental groups were found. More childless women before the deadline ( 12 out of 41 ) and after the deadline (16 out of 51) reported that they never have had a pronounced wish for a child (i.e., wanted a lot to have a child) compared with women who had a child (5 out of 46), $\chi^{2}(2, N=138)=6.51, p<.05$. We examine whether these differences in retrospective childwish account for the differences between the quasi-experimental groups.

\section{Developmental Goals}

The first set of hypotheses addresses the distribution patterns of content categories of developmental goals across different participant groups, dependent on whether they are before the deadline, have passed the deadline, or have successfully met the childbearing deadline. To investigate group differences in the number of nominated developmental goals, a 10 (domain: childbearing, child rearing, occupation, family, finances, health, leisure, self, friends, 
society) $\times 3$ (group: met deadline, missed deadline, urgency) analysis of variance (ANOVA) was performed. The analysis revealed a significant effect for Domain, $F(9,1260)=56.55, p<$ .001 , and a significant Domain $\times$ Group interaction, $F(18$, $1260)=6.89, p<.001$. Further one-way ANOVAs were conducted for each of the 10 goal domains. Significant group effects were obtained for childbearing, $F(2,140)=18.10, p<.001$; child rearing, $F(2,140)=35.76, p<.001$; self-related, $F(2$, $140)=6.23, p<.01$; friends related, $F(2,140)=3.05, p=.05$; and health goals, $F(2,140)=8.42, p<.001$. No significant group effects were found with respect to the number of nominated work, family, leisure, financial, and societal goals. All significant group effects remained stable $(p<.05)$ when controlling for education. We also controlled for retrospective childwish, contrasting women who had a pronounced wish for a child at least for one 5-year period during their adulthood with those women who at no time during their life had a pronounced wish for a child. All group effects for developmental goals remained significant, and the covariate retrospective childwish did not reach significance for any of the goal categories. In addition, we investigated potential age differences within the urgency and the missed-deadline groups, because the younger women in the urgency group might experience less time pressure for childbearing than the older women in the urgency group, and the younger women in the missed-deadline group might still expect to bear a child. The analyses revealed no significant relationship between age and number of childbearing or child-rearing goals in either the urgency or the missed-deadline group (all correlation coefficients smaller than .10).

Table 1 illustrates the mean values and standard deviations of reported developmental goals in the different participant groups for Study 1. Follow-up analyses ( $t$ tests) revealed that women in the urgency group, $t(77.92)=6.77, p<.001$, and women with a child, $t(73.54)=4.63, p<.001$, reported more frequently childbearing-related goals than women who had passed the childbearing deadline. Goals for child rearing were only nominated by women with a child. Women who had passed the deadline reported more frequently self-related and health-related goals than women in the urgency group: self, $t(59.45)=1.99, p=.05$; health, $t(92)$ $=2.64, p=.01$; and women with a child: self, $t(54.16)=2.97, p$ $<.01$; health, $t(67.50)=3.85, p<.001$. With respect to friendsrelated goals, women who had passed the deadline reported more goals than women in the urgency group, $t(72.39)=2.43, p<.05$.

Table 1

Means (and Standard Deviations) of Developmental Goals in Different Groups (Study 1)

\begin{tabular}{lccc}
\hline & \multicolumn{3}{c}{ Group } \\
\cline { 2 - 4 } \multicolumn{1}{c}{ Goal } & Child & Urgent & Just passed \\
\hline Childbearing & $.45(.50)$ & $.61(.49)$ & $.07(.26)$ \\
Child rearing & $.61(.70)$ & $.00(.00)$ & $.00(.00)$ \\
Occupation & $1.00(.65)$ & $1.27(.63)$ & $1.05(.65)$ \\
Family & $.94(.75)$ & $.92(.48)$ & $.70(.60)$ \\
Finances & $.33(.52)$ & $.21(.46)$ & $.28(.45)$ \\
Health & $.10(.31)$ & $.20(.40)$ & $.44(.50)$ \\
Leisure & $.22(.42)$ & $.33(.59)$ & $.37(.62)$ \\
Self & $.14(.41)$ & $.29(.50)$ & $.63(1.00)$ \\
Friends & $.22(.42)$ & $.12(.32)$ & $.32(.47)$ \\
Society & $.04(.20)$ & $.06(.24)$ & $.02(.15)$ \\
\hline
\end{tabular}

\section{Incidental Memory}

The second set of hypotheses implied that individuals show superior recall for information that fits the functional requirements of their motivational phase. Separate dependent variables were created for each category reflecting the number of sentences recalled within that category. Because there were few sentences recalled at a nearly perfect level, the following analyses were performed on sentences recalled either perfectly or on sentences in which the main gist of the sentence was recalled. ${ }^{2}$

We also included a category of neutral (non-baby-related) sentences to control for overall memory differences between the three groups. An ANOVA on the number of neutral sentences recalled revealed no significant differences, $F(2,140)=1.59, p>.20$. This suggests that there were no overall differences in the memory performance of the three groups, so the following analyses were computed on the raw number of sentences recalled within each category. ${ }^{3}$

An overall 10 (category) $\times 3$ (group) ANOVA revealed a significant effect for Group, $F(2,140)=9.20, p<.01$, a significant main effect for category, $F(9,1260)=20.05, p<.01$, and a significant Category $\times$ Group interaction, $F(18,1260)=1.93$, $p<.05$. The main effect for category indicates only that there were differences between categories in number of sentences recalled and is not of interest in the present study. The main effect for group indicates that the three groups differed significantly in the number of baby-relevant sentences that they recalled (note that there were no significant group differences in the number of non-baby-relevant sentences recalled). Follow-up analyses revealed that the urgency group recalled more baby-relevant sentences than did the success group, $t(98)=4.13, p<.01$, and the deadline-passed group, $t(92)=2.83, p<.01$. This finding supports the assumption that baby-relevant materials had an enhanced salience for the urgency group. The Category $\times$ Group interaction is of most interest presently, as it indicates that groups differed in which categories they recalled best and in which categories they recalled least well. This interaction allowed us to examine simple effects of group within each category.

A one-way ANOVA on groups was conducted for each of the 10 categories. Six of the categories revealed effects of group on number of sentences recalled: goal value good, $F(2,140)=3.97$, $p<.05$; goal value bad, $F(2,140)=10.83, p<.01$; positive feedback, $F(2,140)=3.50, p<.05$; attributions that avoid responsibility, $F(2,140)=4.20, p<.05$; competing goals, $F(2$, $140)=3.24, p<.05 ;$ and capitalizing goals, $F(2,140)=7.66$, $p<.01$. In addition, one of the categories contained a marginal effect: substitute goals, $F(2,140)=2.74, p<.10$. When control-

\footnotetext{
${ }^{2}$ Analyses were performed on several levels of recall (complete verbatim, core content, and so on). Results across these levels were highly similar, albeit with minor differences in significance level.

${ }^{3}$ The following analyses were also repeated on the number of sentences recalled within each category, residualized on the number of neutral sentences the individuals recalled. This is a way of statistically removing any differences in general recall ability between individuals. These analyses also revealed highly similar results.
} 
ling for education, all significant group effects remained stable $(p<.05$ ) except for the group effect in competing goals, which was somewhat reduced $(p=.06)$. The covariate analysis for retrospective childwish showed nonsignificant covariate effects and a stable pattern of differences between participant groups.

Specific group comparisons ( $t$ tests) were conducted concerning the significant group effects of the ANOVA results. Figure 2 shows the number of sentences recalled by each group within each category. Participants of the urgency group recalled more sentences in the categories goal value bad, positive feedback, competing goals, and capitalizing goals compared with the postdeadline group (all $t \mathrm{~s}>2.0$, all $p \mathrm{~s}<.05$ ) and to the success group (all $t \mathrm{~s}>2.20$, all $p \mathrm{~s}<.05$ ). Moreover, with regard to the categories goal value good and competing goals, participants of the urgency group reported higher ratings than women who had a child (all $t \mathrm{~s}>2.30$, all $p \mathrm{~s}<.05$ ). Finally, women who missed the deadline recalled more sentences in the category attribution (avoid responsibility) compared with women with a young child, $t(63.54)=2$. $64, p=.01$.

To determine whether the younger women on the urgency group exhibited urgency-related enhanced recall for child-relevant sentences and whether younger women in the passed-deadline group showed disengagement-related recall, we investigated age differences within the urgency and missed-deadline groups. Separate analyses on recalled sentences were conducted for these two groups. In the urgency group, younger women recalled more sentences about means and opportunities $(r=-.42, p<.01)$ and positive feedback about childbearing $(r=-.38, p<.01)$, as well as about competing goals $(r=-.34, p=.014)$ and capitalizing goals $(r=.34, p<.016)$. This implies that the younger women in the urgency group showed at least as much and even somewhat enhanced recall for child-relevant sentences compared with older women in this group. In the missed-deadline group, younger women recalled fewer sentences about positive goal value of having a child $(r=.40, p<.01)$ and more sentences about substitute goals ( $r=-.45, p<.01$ ). Thus, younger women in the passed-deadline group exhibited a recall pattern even more pronouncedly disengaging from childbearing than the older women in this group.

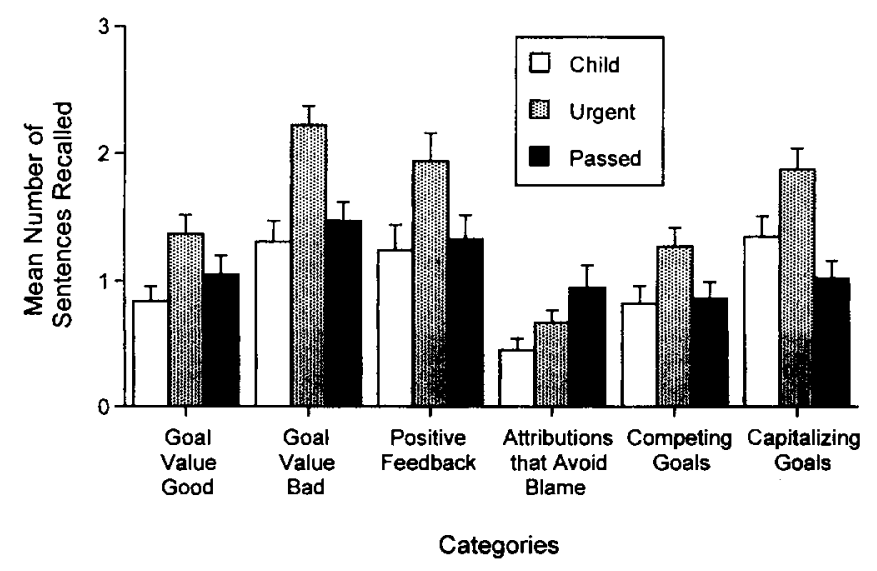

Figure 2. Mean number of sentences recalled by three groups of participants in the incidental memory task.

\section{Relationships Between Recall and Well-Being}

The rationale underlying our hypotheses was as follows. Motivational phases differ in their functional requirements, which in turn render certain kinds of information more relevant than other types of information. Thus, information processing to be adaptive for a given motivational phase should be biased toward enhancing the salience of information functionally relevant for a given motivational phase and decreasing the salience of irrelevant or even conflicting information. This is a general model of how motivational phases should affect information processing in groups of participants differing in their pre- and postdeadline status.

The results concerning recall performance (see section above) did indeed show that individuals from different deadline-relevant groups showed differential recall for phase-relevant content categories of sentences. However, the adaptiveness of the information processing can also be investigated in terms of interindividual differences in matching information processing to the respective motivational phase. The purpose of this section of results is to test the general hypothesis that, within a given motivational phase, those individuals who recall more sentences relevant to the functional requirements of their motivational phase should also report higher well-being.

Table 2 shows correlations between number of sentences recalled within each category and the two well-being measures of positive and negative affect separately for each group. Positive affect and negative affect were chosen as well-being measures because research has demonstrated positive and negative affect to be differentially related to failure and success (e.g., Warr, Barter, \& Brownbridge, 1983; Watson et al., 1988). Considering first the passed-deadline group, there is an overall pattern of positive correlations between number of sentences remembered and negative affect: Those individuals who missed the deadline but nonetheless recalled many sentences about babies reported the greater levels of negative affect. Five of these categories were particularly strongly associated with experience of negative affect: goal value good, goal value bad, attributions that avoid responsibility, attributions that take responsibility, and capitalizing goals. In regard to positive affect, two significant correlations were found for the passeddeadline group: Substitute goals was related to enhanced experience of positive affect, and goal value good was correlated with lower levels of positive affect. In contrast, recall of sentences was not associated with either positive affect or negative affect for the groups of participants who were in the urgency or met-deadline phases. The only exception was a significant correlation among those in the met-deadline phase between negative affect and the number of sentences recalled in the age-timing category. We obtained the same pattern of correlations between incidental memory and affect in the three groups when controlling for education. The pattern of partial correlations also remained stable when controlling for retrospective childwish.

In summary, the exploratory hypothesis about the predictive relationship between phase-adequate selective recall and affect yielded a model-consistent pattern of findings. For the misseddeadline group, negative affect was consistently associated with recall of child-relevant sentences, almost irrespective of the sentences' specific content. In addition, positive affect of misseddeadline women was related to high recall of substituting goals and depressed recall of the positive value of having children. 
Table 2

Correlations Between Number of Sentences Recalled Within Each Content Category and Participants' Positive Affect (PA) and Negative Affect (NA)

\begin{tabular}{|c|c|c|c|c|c|c|}
\hline \multirow[b]{2}{*}{ Content category of sentence recalled } & \multicolumn{2}{|c|}{ Met deadline } & \multicolumn{2}{|c|}{ Urgent } & \multicolumn{2}{|c|}{ Passed deadline } \\
\hline & PA & NA & PA & NA & PA & NA \\
\hline Goal value good & -.03 & .18 & .17 & .18 & $-.35 *$ & $.37^{*}$ \\
\hline Goal value bad & .14 & .03 & .16 & .11 & -.09 & $.38^{*}$ \\
\hline Means and opportunities & .16 & -.12 & .23 & -.13 & -.03 & -.06 \\
\hline Feedback (positive) & .22 & -.04 & .20 & -.17 & .12 & .05 \\
\hline Attributions that avoid responsibility & -.01 & .17 & -.03 & .20 & -.16 & $.44^{* *}$ \\
\hline Attributions that take responsibility & .05 & .12 & .02 & .14 & -.17 & $.43^{* *}$ \\
\hline Age timing & -.08 & $.29 *$ & .01 & -.13 & -.29 & .11 \\
\hline Competing goals & .20 & .01 & .12 & -.22 & .00 & .14 \\
\hline Substitute goals & .14 & .03 & .14 & -.11 & $.37 *$ & .14 \\
\hline Capitalize on goal attainment & -.11 & .22 & .05 & -.11 & -.15 & $.41 * *$ \\
\hline
\end{tabular}

${ }^{*} p<.05 . \quad{ }^{* *} p<.01$.

\section{Discussion}

The results of Study 1 provide support to the general hypotheses raised in the introduction. Women approaching the developmental deadline for childbearing are actively engaged with the goal of bearing a child. In contrast, those women who have passed the deadline disengage from the goal of childbearing. Specifically, Study 1 provided evidence with regard to two types of indicators of goal orientations: (a) developmental goals nominated and (b) salience of information reflected in incidental recall.

The findings pertaining to the developmental goals nominated by the participants reflect the contrasting opportunities for the different groups of women studied here. Predeadline women without a child, the urgency group, reported the most goals related to bearing a child and fewer goals in all other goal categories than women who had passed the deadline. For this group apparently childbearing had become the most pressing developmental goal. In contrast with this urgency pattern, childless women who had passed the deadline reported almost no goals directed at childbearing and focussed instead on developing their self, their social network, and their health. Women with children seemed to capitalize on their attained parenthood and expressed a focussed investment in family life.

The findings about group-differential recall of baby-relevant sentences did not match the predicted pattern of selectively enhanced recall for sentences consistent with pre- or postdeadline motivational orientation, respectively. A more crude and in a sense more radical pattern was found. Women in the urgency group exhibited superior recall of almost all types of baby-relevant sentences when compared with the other groups. For the interpretation of this finding it is important to keep in mind that no group differences were found for the recall of neutral sentences, so that differences in recalling specific content categories cannot be attributed to general group differences in recall ability. Moreover, the urgency effect was not restricted to women in their early $30 \mathrm{~s}$ but pertained to women in their late 20 s too. Indeed, there was some evidence that younger women in the urgency group were even more keyed into child-relevant information. Analogously, younger women compared with older women in the passeddeadline group appeared to be more receptive of disengagement- related sentences. Further research on this phenomenon might provide convergent evidence for enhanced cognitive receptiveness in the beginning of critical phases in life (i.e., deadline approaching and deadline passed) as contrasted with the mind set of older individuals who have grown accustomed and tuned to the life phase they are in.

This finding of superior recall for baby-relevant sentences in the urgency group suggests that urgent goal orientations may be associated with a generally enhanced receptiveness to goal-relevant information, regardless of its valence pro or contra goal striving. This implies that the phase-congruent receptiveness relies on surface cues rather than on deep processing of sentence meaning.

The only exception to the urgency pattern of recalling babyrelevant information was sentences about avoiding self-blame for childlessness, which were better recalled by women who had passed the childbearing deadline. This enhanced recall is possibly related to the specific meaning of the sentences, which is conducive to self-protection in this group of irreversibly childless women.

In addition to the congruency between pre- and postdeadline developmental potential and goal orientation (engagement vs. disengagement), we also investigated the association of this phase congruency and emotional well-being. Interestingly, in this regard the findings also point to a general receptiveness to baby-relevant cues as the critical feature. Women who had passed the deadline, and who showed superior recall of various categories of babyrelevant sentences, were more prone to negative affect. Thus, a selective receptiveness to information relevant for an obsolete developmental goal appears to have negative implications for psychological well-being. Salience of child-related information might activate ruminations (Nolen-Hoeksema, Parker, \& Larson, 1994) about the lost opportunity of having children, and thus lead to enhanced negative affect or even symptoms of depression. We also found evidence for content-specific effects on affect. Positive affect in passed-deadline women was associated with recall of substitute goals and depressed recall of the positive value of having children. Facilitated processing for alternative goals to childbearing and inhibited processing of its benefits probably enables the individual to attend to other domains of functioning 
and to invest her resources productively in goal pursuits that are not futile.

\section{Study 2}

Study 1 had confirmed different motivational orientations in pre- and postdeadline women. However, we did not find evidence for the usage of specific control strategies in pre- and postdeadline individuals, because the indicator used, sentence recall, proved to be only partially sensitive to the specific sentence content.

The major aim for Study 2 was, therefore, to directly assess control strategies by asking participants explicitly whether they use these strategies in the context of goals for childbearing. This allows us to investigate whether the assumptions that the model of developmental regulation around deadlines makes in terms of control strategies conducive to predeadline goal engagement and postdeadline goal disengagement are valid. Study 2 extended and supplemented the paradigm of Study 1 in three ways.

First, we included a questionnaire about primary and secondary control strategies for the domain of wanting to have a child, which is based on the domain-general OPS Scales (J. Heckhausen, Schulz, \& Wrosch, 1998). Second, a group of pregnant women (beginning the third trimester of pregnancy) was included in the sample design. Studying pregnant women was interesting to us because they are close to achieving the goal of having a child without actually living with a child. Of particular interest was the subtle but, as we believe, important difference between women in the urgency and in the pregnant condition. Women who are 6 months pregnant can be almost certain to succeed in having a child, whereas urgency itself can bring about ambivalent goal striving because of the failure anxiety that comes with uncertain success potential. The latter assumption was already suggested by the finding of Study 1 that women in the urgency condition more frequently recalled negative aspects of living with a child and competing goals to childbearing. Finally, we also introduced a group of women who, on the basis of their age in the early $50 \mathrm{~s}$, can be assumed to be long passed the childbearing deadline. This group is interesting because it allows us to investigate whether compensatory secondary control strategies of goal distancing and self-protection are only needed shortly after the deadline is passed or whether goal engagement and threats to self-esteem prevail across extended periods of time after the deadline. Our expectation is that compensatory secondary control is particularly activated just after passing the deadline and thereafter becomes continuously less needed for adaptive functioning and emotional well-being.

\section{Method}

\section{Participants}

Participants were recruited by not only using similar strategies as were used in Study 1 but also by contacting pregnant women the interviewers found by visiting prenatal courses at maternity hospitals. Participants $(N=$ 222) were invited to participate if they met the criteria for one of the following five criteria. Just passed deadline if they were age 39 to 46 years $(n=49, M=42.44$ years, $=1.98)$ and childless, long passed deadline if they were 49 to 56 years $(n=30, M=52.50$ years, $S D=1.80)$ and childless, met deadline if they were age 18 to 41 ( $n=50, M=30.98$ years, $S D=5.06)$ and their only child was less than 1 year old, pregnant if they were age 21 to 39 years $(n=46, M=28.85$ years, $S D=4.27)$ and were pregnant in the beginning of the third pregnancy trimester, and urgency if they were age 29 to 35 years $(n=47, M=32.13$ years, $S D=1.58)$ and had never had a child. Sixty-three percent $(n=139)$ of the women were highly educated (German Abitur and higher). This proportion of highly educated participants makes the sample very similar to the sample of Study 1, which had involved $63 \%$ with high education. As in Study 1, participants received DM 40 (about $\$ 23$ ) for their participation.

\section{Procedure and Materials}

The procedure was similar to Study 1. Groups of 5 to 6 participants were invited to the Max Planck Institute. After a warm-up phase, participants were requested to complete a questionnaire including OPS Scales, developmental goals, depressive symptoms, sociodemographic characteristics, and retrospective childwish. Sessions lasted approximately $90 \mathrm{~min}$.

A new measurement instrument for primary and secondary control strategies (OPS Scales; J. Heckhausen et al., 1998) was adapted to the specific domain of childbearing. Previous research has confirmed domainspecific indicators of control striving to effectively assess differences in developmental regulation of specific goals (Schulz, Wrosch, Yee, Heckhausen, \& Whitmer, 1998; Wrosch \& Heckhausen, 1999). Participants were asked to indicate how true each statement was for them on a 5-point scale, ranging from $1=$ not true at all to $5=$ very true. These OPS Scales included subscales (four items per scale) of selective primary control (exemplar item: "I try everything possible to have own children," Chronbach's $\alpha=.87$ ), selective secondary control (exemplar item: "I try not to be distracted by other things from my wish for a child," $\alpha=.83$ ), compensatory primary control (exemplar item: "When having a child proves difficult for me, I seek the advice of others (e.g., physicians)," $\alpha=$ .87 ), and compensatory secondary control (exemplar item: "If I can not realize my wish for a child, I can get it out of my mind," $\alpha=.39$ ). The reliability of the Compensatory Secondary Control scale was somewhat low. However, it should be noted that the Compensatory Secondary Control scale includes different subsets of strategies (i. e., self-protection, goal disengagement; Wrosch \& Heckhausen, 1999) that are functionally complementary but therefore also may serve as substitutes for each other in different individuals, thus bringing about a relatively low scale consistency.

On the basis of the results from Study 1 that showed control orientation to be related to negative affect in passed-deadline participants, we also assessed depressive symptoms by using the 20 -item Center for Epidemiological Studies Depression Scale (CES-D; Radloff, 1977). Participants were asked to indicate how often each statement (e.g., felt lonely, felt depressed, felt that everything was an effort) applied to them during the past weeks on a 4-point scale, ranging from $0=$ rarely or none of the time to $3=$ most or almost all of the time. For this sample, a Cronbach's alpha of $.90(M=12.29, S D=9.51)$ was obtained. In addition, we assessed participants' developmental goals by using the same instrument as described in Study 1 .

\section{Results}

The results of Study 2 are divided into three sections. First, we examined participants' developmental goals. Obtaining comparable group differences in a second, unrelated sample is expected to provide information about the generalizability of the phenomenon. Second, we investigated whether the participants of the five groups reported a phase-congruent endorsement of primary and secondary control strategies. Third, we examined whether reported control strategies predict depressive symptoms in childless women, depending on whether they have or have not passed the childbearing deadline. As to the variable retrospective childwish, differences between the five groups were obtained, as expected, $\chi 2(4, N=$ 
$222)=22.70, p<.01$. The numbers of women in the five groups who reported to never have had a pronounced childwish were 5 (out of 50 ) in the group of women with a child; 8 (out of 47) in the urgency group; 17 (out of 49) in the just-passed-deadline group; 14 (out of 30) in the long-passed-deadline group; and 9 (out of 46) in the group of pregnant women.

\section{Developmental Goals}

To investigate group differences in the number of nominated developmental goals, a 10 (domain: childbearing, child rearing, occupation, family, finances, health, leisure, self, friends, society) $\times 5$ (group: urgency, pregnant, child, just passed, long passed) ANOVA was performed. The analysis revealed a significant effect for domain, $F(12,2604)=95.64, p<.001$, and a significant Domain $\times$ Group interaction, $F(48,2604)=6.10, p<.001$. Further one-way ANOVAs were conducted for each of the 10 goal domains. Significant group effects were obtained for childbearing, $F(4,217)=41.95, p<.001$; child rearing, $F(4,217)=21.07, p<$ .001 ; family, $F(4,217)=4.17, p<.01$; health, $F(4,217)=15.34$, $p<.001$; leisure, $F(4,217)=5.87, p<.001$; self, $F(4$, $217)=3.95, p<.01$; and friends, $F(4,217)=8.60, p=.05$. All group effects were stable $(p<.05)$ when controlling for participants' education. When introducing the covariate retrospective childwish into the analyses, all group differences remained significant; for only the goal category childbearing the covariate reached significance, $F(1,216=7.72 ; \beta=.147, p<.01$.

Table 3 shows the group differences in nominated developmental goals. Similar to the results of Study 1, women with a child, $t(85.74)=5.65, p<.001$, and women in the urgency group, $t(77.54)=7.20, p<.001$, reported more childbearing goals than women who had just passed the deadline. Pregnant women and women of the long-passed-deadline group reported no childbearing goals. In addition, women with a child reported more childrearing goals than women of the four other groups: urgency, $t(66.32)=4.47, p<.001$ (other groups did not mention childrearing goals). With regard to the family domain, just-passeddeadline women reported fewer goals than pregnant women, $t(93)=-2.53, p<.05$. Moreover, women of the long-passeddeadline group reported fewer family goals compared with pregnant women, women with a child, and urgent women (all $t \mathrm{~s}<$ -2.60 , all $p s<.05$ ). For the health domain, more goals were nominated by pregnant women and long-passed-deadline women as compared to women with a child, urgent women, and justpassed-deadline women (all $t \mathrm{~s}>2.50$, all $p \mathrm{~s}<.001$ ). With regard to leisure, pregnant women mentioned fewer goals than urgent women, just-passed-deadline women, and long-passed-deadline women (all $t \mathrm{~s}<-2.50$, all $p \mathrm{~s}<.01$ ). Moreover, just-passeddeadline women reported more leisure goals than women with a child and urgent women (all $t \mathrm{~s}>2.10$, all $p \mathrm{~s}<.05$ ). Self-related goals were more frequently mentioned by women of the justpassed-deadline group as compared to women with a child and urgent women (all $t \mathrm{~s}>2.70$, all $p \mathrm{~s}<.01$ ). In addition, women who recently gave birth to a child reported fewer self-related goals than pregnant women, $t(94)=-2.47, p<.05$. Finally, friendsrelated goals were more frequently reported by long-passeddeadline women as compared with women with a child, pregnant women, urgent women, and just-passed-deadline women (all $t \mathrm{~s}>2.70$, all $p \mathrm{~s}<.01$ ). In addition, women who had just passed the deadline reported more friends-related goals than did pregnant women, $t(62.11)=2.70, p<.01$.

To investigate age differences within the urgency and the justpassed-deadline group, separate analyses were conducted. Within the just-passed-deadline group no age differences in goal nomination pattern were identified (all correlations with age smaller than $.10)$. Within the urgency group younger women reported fewer goals pertaining to leisure activities $(r=.30, p<.05)$ and friendships $(r=.46, p<.01)$ than older women. Thus, younger women in the urgency group were at least as engaged with childrelated goals as were older women in this group.

\section{Control Strategies}

To examine group differences in the endorsement of control strategies, we conducted a 4 (strategy: selective primary, selective secondary, compensatory primary, and compensatory secondary control) $\times 5$ (group: urgency, pregnant, child, just passed, long passed) ANOVA. We found significant effects for group, $F(4$, $217)=21.13, p<.001$; strategy, $F(3,651)=14.78, p<.001$; and the Group $\times$ Strategy interaction, $F(12,651)=15.71, p<$ .001 . Further one-way ANOVAs showed significant group effects for selective primary control, $F(4,217)=31.31, p<.001$; selective secondary control, $F(4,217)=27.01, p<.001$; compensatory primary control, $F(4,217)=6.25, p<.001$; and

Table 3

Means (and Standard Deviations) of Developmental Goals in Different Groups (Study 2)

\begin{tabular}{lrrrrr}
\hline & \multicolumn{5}{c}{ Group } \\
\cline { 2 - 6 } \multicolumn{1}{c}{ Goal } & Child & Pregnant & Urgent & Just passed & Long passed \\
\hline Childbearing & $.60(.49)$ & $.00(.00)$ & $.77(.52)$ & $.12(.33)$ & $.00(.00)$ \\
Child rearing & $.38(.49)$ & $.00(.00)$ & $.04(.20)$ & $.00(.00)$ & $.00(.00)$ \\
Occupation & $1.18(.63)$ & $1.50(.91)$ & $1.28(.62)$ & $1.31(.71)$ & $1.10(.71)$ \\
Family & $.82(.60)$ & $1.00(.67)$ & $.94(.76)$ & $.69(.51)$ & $.47(.57)$ \\
Finances & $.26(.44)$ & $.28(.54)$ & $.21(.41)$ & $.18(.39)$ & $.20(.41)$ \\
Health & $.36(.52)$ & $1.09(.78)$ & $.34(.52)$ & $.57(.64)$ & $1.10(.61)$ \\
Leisure & $.24(.48)$ & $.09(.28)$ & $.30(.46)$ & $.53(.62)$ & $.47(.57)$ \\
Self & $.30(.65)$ & $.67(.82)$ & $.40(.68)$ & $.82(.81)$ & $.50(.63)$ \\
Friends & $.12(.36)$ & $.02(.15)$ & $.13(.34)$ & $.18(.39)$ & $.50(.57)$ \\
Society & $.10(.36)$ & $.04(.21)$ & $.06(.32)$ & $.04(.20)$ & $.17(.46)$ \\
\hline
\end{tabular}


compensatory secondary control, $F(4,217)=6.84, p<.001$. Group effects remained significant when education was controlled. Entering the covariate retrospective childwish also did not alter the pattern of group differences. However, the covariate reached significance for selective primary control, $F(1,216)=25.62, \beta=$ $.273, p<.01$; selective secondary control, $F(1,216)=25.18, \beta=$ $.279, \quad p<.01 ;$ and compensatory primary control, $F(1$, $216)=12.12, \beta=.230, p<.01$. In addition, separate analyses for age differences in the urgency and the just-passed-deadline groups showed no age effect in either group (correlations with age were smaller than .20).

Figure 3 illustrates the group differences in the endorsement of the four control strategies. As expected, follow-up analyses $(t$ tests) revealed that women of the urgency group, pregnant women, and women with a child reported higher levels of selective primary control, $t(220)=11.08, p<.001$; selective secondary control, $t(220)=10.46, p<.001 ;$ and compensatory primary control, $t(220)=4.73, p<.001$; than women who had just or long ago passed the deadline for childbearing. In contrast, women who had passed the childbearing deadline (just passed and long passed) reported higher ratings in compensatory secondary control, $t(220)=4.94, p<.001$, than women of the three other groups. No differences in reported control striving were obtained between just-passed- and long-passed-deadline women, as well as between women of the urgency group, the pregnant group, and the child group.

\section{Predicting Depressive Symptoms in Childless Women}

To test our hypotheses about the adaptive value of a phasecongruent endorsement of control strategies, we performed regression analyses for predicting depressive symptoms (using the CES-D) in childless women who had not passed the childbearing deadline (urgency group) and women who had already passed the deadline (just-passed- and long-passed-deadline groups). First, we tested the main effects of reported control striving and the group variable. The group variable contrasted childless women before the

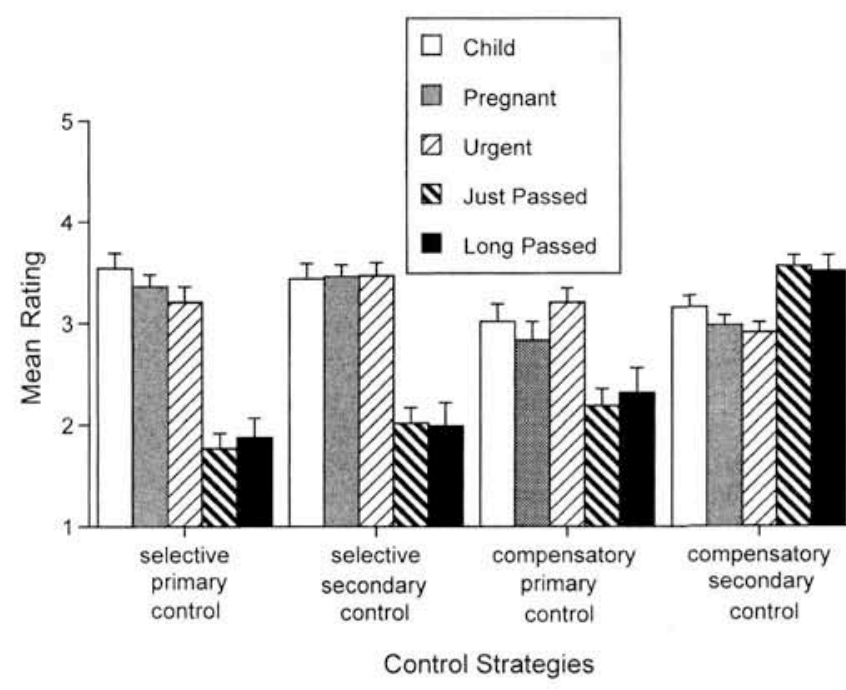

Figure 3. Endorsement of primary and secondary control strategies by five groups of participants. deadline with childless women after the deadline. We then examined the hypothesized interactions of control processes with the group variable for significance. We also controlled the results for participants' education and retrospective childwish. Both were unrelated to level of depressive symptomatology and did not affect the pattern of findings reported below. We then excluded nonsignificant predictors from the regression equations.

We obtained a significant main effect for selective secondary control. Participants who reported higher levels of selective secondary control showed more depressive symptoms, $F(1,125)$ $=6.09, \beta=.49, p=.01$. In addition, we found a significant interaction effect between selective primary control and the group variable for predicting depressive symptoms, $F(1,125)=7.25$. $p<.001$. Both the main effect and the interaction effect remained significant when education and the other control strategies were controlled.

To illustrate the significant interaction effect, Figure 4 displays the regression of selective primary control on depression for both groups of childless women. As expected, women who had passed the childbearing deadline showed higher levels of depressive symptoms if they reported greater selective primary control. In contrast, the endorsement of selective primary control reduced the risk of developing depressive symptoms in childless women who had not yet passed the deadline. It should be mentioned that we also found a significant interaction between selective secondary control and participants' group that shows selective secondary control to be positively related to depression in passed-deadline women $(r=.31, p<.05)$ but unrelated to depression in predeadline women $(r=-.14, p>.10)$. However, the interaction effect between selective secondary control and participants' group failed to reach significance when controlling for the other control strategies.

To obtain more specific information about whether the effect of selective primary control on depressive symptoms depends on the age of passed-deadline women, we computed partial correlations for only passed-deadline women between selective primary control and depressive symptoms, simultaneously controlling for age and age-squared (possible quadratic effect). The correlation between selective primary control and depressive symptoms $(r=.26, p<$ $.05)$ did not change when controlling for age and age-squared ( $r=$ $.27, p<.05$ ), indicating that the negative effect of selective primary control on depressive symptoms is not related to the age of passed-deadline women.

\section{Discussion}

The major aim of Study 2 was to demonstrate differential use of control strategies directly assessed by self-report in predeadline and postdeadline individuals. In addition, we assessed developmental goals and expected to replicate the findings of pre-versus postdeadline differences obtained in Study 1. Moreover, two additional groups were included to investigate the generalizability of findings about predeadline functioning from an urgency condition to the condition of pregnancy and about postdeadline functioning from women who had just passed to those who had long passed the deadline.

The comparison of nominations for developmental goals across the two studies reveals a consistent pattern of group differences. Predeadline women and the women with children were engaged in 


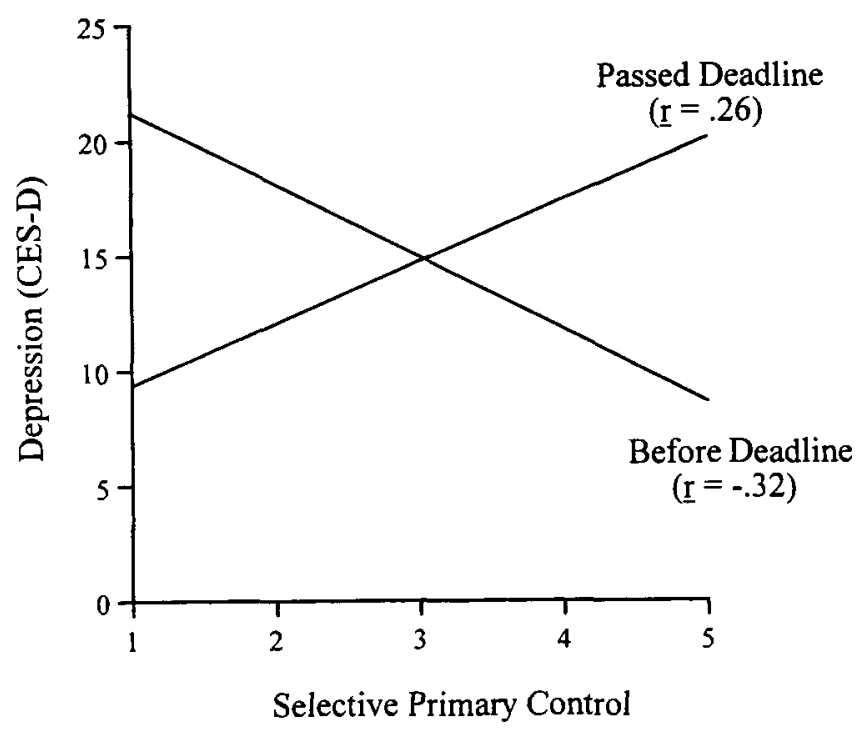

Figure 4. Selective primary control as predictor of depressive symptoms in childless women who have and have not passed the childbearing deadline. CES-D = Center for Epidemiological Studies Depression Scale.

goals of childbearing, child rearing (only women with child), and other family issues, whereas women who had passed the deadline recently or long ago were engaged with other nonfamily goals. To avoid misunderstandings, it should be emphasized that the greater number of nonfamily goals nominated by women in the passeddeadline groups should not be viewed as mere substitutes of family life. Instead, women without children may have had more opportunities to accumulate social resources outside the family, in particular with regard to vocational fulfillment and social networks.

The findings about the pregnant women reveal a special position for this group. Not surprisingly childbearing goals are absent in this group. These women are so far advanced in their pregnancies that this goal is almost reached. However, the pregnant women also did not mention child-rearing goals as one might have expected, given that they would be giving birth within the next 3 months. It may be that pregnancy is such a salient and potentially risky developmental phase that future goals about the child going beyond the birth are pushed out of the reach of current planning.

The results pertaining to the specific control strategies show a clear contrast between the three pre-deadline and deadline-met groups on the one hand and the two post-deadline groups on the other hand. The three kinds of control strategies involved in goal engagement (selective primary, selective secondary, and compensatory primary control) were rated higher by the women with a child, the pregnant women, and the women just before the deadline. In contrast, compensatory secondary control strategies involving goal disengagement and self-protection were more valued by the women who had recently or a long time ago passed the deadline. We must put a caveat, however, on the interpretation of findings about compensatory secondary control because of the low internal consistency of the scale. This low reliability may be due to the fact that compensatory secondary control comprises two components, goal disengagement and self-protection, which, albeit complementary, may have different functionality for certain situations. Urgent predeadline individuals, for instance, might well require self-protective strategies of compensatory secondary control after a failed attempt to reach a goal, but would be ill advised to disengage from the goal prematurely.

Finally, the analyses about the phase adequacy of control strategies as a predictor of depressive symptoms supported and extended the findings from Study 1, both in terms of the measurement instrument used to identify control strategies and in terms of the indicator of subjective well-being. Selective primary control strategies were associated with less depressive symptomatology in the urgency group, and with an enhanced risk of depression in the groups who had passed the deadline without having a child. Given that the study is cross-sectional, the causal sequence remains unclear; possibly depressed women tended to hang on to and ruminate about the obsolete childbearing goal more than did nondepressed women. However, a greater tendency for selective primary control, albeit for a futile goal, is not a typical feature of depressive behavior. Moreover, longitudinal research about engagement with health maintenance goals has revealed predictive relations of goal engagement control strategies and lower levels of depression (Wrosch, Schulz, \& Heckhausen, 2000). Thus, it seems more plausible to interpret the causal sequence as being led by selective primary control striving for an unattainable goal, which then leads to continuous frustration and control loss, and eventually depression. Thus, it can be concluded that phase congruency of control strategies promoted mental health and subjective wellbeing in Study 1 and in Study 2.

In addition, it was found that phase-incongruent selective primary control striving was detrimental of mental health for all women who had passed the deadline, irrespective of their age and thus of how long ago the deadline had been passed. Whatever tendencies for wanting a child were left even long after passing the deadline, they were as detrimental to mental health as in the group who had just recently passed the deadline. This is surprising, because one might have expected a "cooling out" of such effects in the long-passed-deadline group in the sense that these women in their early $50 \mathrm{~s}$ should have overcome the struggles of disengaging from childbearing goals. It seems, however, that they did not, and the question is whether this is a sample-specific finding or whether childbearing goals in general are difficult to deactivate because they represent a major developmental task (Havighurst, 1952). Moreover, childless women may experience a continued or revitalized feeling of regret about their childlessness when reaching the age of grandparenthood (Alexander, Rubinstein, Goodman, \& Luborsky, 1992), so that long obsolete states of goal orientation toward childbearing are reactivated in late midlife.

\section{General Discussion}

In the general discussion, we consider first the limitations of the present research and then discuss the implications of our findings and potential future research.

\section{Limitations}

We chose a quasi-experimental approach to investigate individuals' developmental regulation around deadlines for developmental goals. By studying the goal of childbearing and groups of women whose age puts them before rather than after the deadline for childbearing, we chose an ecologically valid, very important, 
and thus motivationally involving paradigm. However, this choice also involves potential problems of confounding initial group differences with differences due to having or not having passed the deadline.

A serious potential confound is differential motivation to have a child, irrespective of one's deadline status. This confound was addressed by investigating the effect of having had versus not having had the wish for a child at some time during adulthood. This variable has its problems, of course, since retrospective childwish itself may be reconstructed on the basis of current developmental status. However, such reconstructions would have rendered the covariate tests more conservative in that they would have accentuated differences between groups and thus yielded a stronger effect for the covariate.

We found that the groups differed in the number of participants who reported to never have had a pronounced wish for a child. These differences would have been expected, partly as a product of biased biographical reconstruction. However, these differences were shown to not account for the group differences found with regard to goal orientation (except for the childbearing goal itself in Study 2), control strategies, incidental memory, and predictive relations of control strategies or incidental memory with psychological well-being. Thus, although biased reconstructions were probably yielding larger group differences in retrospective childwish, the patterns of differences between groups were stable against this conservative test. Moreover, we did find that selective recall and control strategies associated with goal engagement for childbearing had negative effects on mental health in women who were either just passed or long passed the deadline. This should not have been the case, if these women had not ever been involved in childbearing goals and thus would have been invulnerable to ruminations and phase-inadequate control tendencies.

Finally, the findings about selective receptiveness to information about childbearing in the pre- and postdeadline women need to be interpreted cautiously. The women participating in the two studies underwent a warm-up procedure, which rendered salient the issue of having or not having a child. Participants in all groups did this warm-up phase, so that the group differences in sentence recall and their relations with affect should not be affected by this procedure, except for rendering their parental status more salient. Thus, one cannot conclude that these women would have shown similar selective recall in a setting outside the laboratory without being mentally set up for the issue of childbearing. Moreover, even if the warm-up procedure did enhance the group differences between parents and nonparents, within-group differences in recall should not have been affected, and neither their association with affect.

\section{Conclusion and Perspectives for Future Research}

In the present research we investigated individuals' developmental regulation before a developmental deadline, after a developmental deadline, and when in the process of or after having met a deadline. In particular, we studied the deadline of having a child. We chose this developmental task and deadline for three primary reasons. First, it is a widely pursued task, providing for less variability across individuals in their evaluation of this task than there would be variability in evaluation of other tasks. Second, the deadline for this task is relatively uncontrollable, relatively nonnegotiable, and relatively narrow in terms of the number of years it spans. Specifically, few individuals have children after their early $40 \mathrm{~s}$. Third, this is a task studied in other contexts and areas of psychology because of its inherent interest. However, the primary purpose of this research was not to investigate childbearing in and of itself. Rather, the main intended contribution of this article is to further our understanding of developmental regulation around important life-course transitions.

The findings of the two studies support our theoretical claim that developmental regulation is organized in action phases, which are timed by the age-graded structure of opportunities for attaining developmental goals. Developmental deadlines appear to function as timing scaffolds for the individual to invest control resources into intense and urgent goal striving when approaching the deadline. Moreover, passing a developmental deadline unsuccessfully may serve as a signal to activate compensatory secondary control directed at goal disengagement and self-protection. In addition to supporting the expected pattern of phase-congruent control behaviors, the two studies also provide evidence for their effects on psychological well-being. Phase-congruent control strategies and information-processing biases were found to be protective against negative affect and depressive symptoms and conducive to positive affect.

The developmental deadline studied in this research is prototypical for the deadline concept in that it is relatively short term, low in controllability, and comparatively high in degree of irrevocability of the childbearing deadline. However, there probably are developmental deadlines that are more mutable and longer term than the deadline associated with the biological clock. This holds, for example, for the timing of marriage (Modell, 1980; Modell, Furstenberg, \& Strong, 1978) or the timing of career transitions (at least when aggregated across career tracks). The action-phase model of developmental regulation argues that deadlines are conditions that represent a change from greater and richer opportunities to lesser and weaker opportunities. Such changes do not need to be absolute or irrevocable but are likely to induce shifts in goal engagement and the activation of control strategies. Recent research on goal engagement and disengagement in the partnership domain, for instance, demonstrates that even with less irrevocable and more continuous age gradients of control loss (marriage and remarriage probabilities), pre- and postdeadline patterns of goal engagement and control behavior can be identified (Wrosch, 1999; Wrosch \& Heckhausen, 1999). Future research may exploit the action-phase model of developmental regulation to study control processes involved in age-graded transitions in various domains of life, be it education, family, work, or health.

To reach its full potential as a research paradigm to study dynamic processes of regulation, research about developmental deadlines should use longitudinal designs to track the microsequential change of goal engagement and disengagement and the respective control strategies in individuals with initially similar goal priorities. This kind of longitudinal research is currently underway with regard to the long-term tracking of control behavior in adults with acute and chronic health problems (Schulz et al., 1998) and with respect to the transition of adolescents from school to work (J. Heckhausen \& Tomasik, in press). 


\section{References}

Alexander, B. B., Rubinstein, R. L., Goodman, M., \& Luborsky, M. (1992). A path not taken: A cultural analysis of regrets and childlessness in the lives of older women. The Gerontologist, 22, 618-626.

Cook, T. D., \& Campbell, D. T. (1979). Quasi-experimentation: Design and analysis for field settings. Chicago: Rand McNally.

Gollwitzer, P. M. (1990). Action phases and mind-sets. In E. T. Higgins \& R. M. Sorrentino (Eds.), Handbook of motivation and cognition: Foundations of social behavior (Vol. 2, pp. 53-92). New York: Guilford Press.

Gollwitzer, P. M., Heckhausen, H., \& Steller, B. (1990). Deliberative and implemental mind-sets: Cognitive tuning toward congruous thoughts and information. Journal of Personality and Social Psychology, 59, $1119-1127$.

Havighurst, R. J. (1952). Developmental tasks and education. New York: McKay.

Heckhausen, H. (1991). Motivation and action. New York: Springer.

Heckhausen, H., \& Gollwitzer, P. M. (1987). Thought contents and cognitive functioning in motivational and volitional states of mind. Motivation and Emotion, 11, 101-120.

Heckhausen, J. (1997). Developmental regulation across adulthood: Primary and secondary control of age-related challenges. Developmental Psychology, 33, 176-187.

Heckhausen, J. (1999). Developmental regulation in adulthood: Agenormative and sociostructural constraints as adaptive challenges. New York: Cambridge University Press.

Heckhausen, J., \& Schulz, R. (1993). Optimisation by selection and compensation: Balancing primary and secondary control in life-span development. International Joumal of Behavioral Development, 16, 287-303.

Heckhausen, J., \& Schulz, R. (1995). A life-span theory of control. Psychological Review, 102, 284-304.

Heckhausen, J., Schulz, R., \& Wrosch, C. (1998). Developmental regulation in adulthood: Optimization in primary and secondary control-A multiscale questionnaire (Tech. Rep.). Berlin, Germany: Max Planck Institute for Human Development and Education.

Heckhausen, J., \& Tomasik, M. (in press). Get an apprenticeship before school is out: How German adolescents adjust vocational aspirations when getting close to a developmental deadline. Journal of Vocational Behavior.

Modell, J. (1980). Normative aspects of American marriage timing since World War II. Journal of Family History, 5, 210-234.

Modell, J., Furstenberg, F. F., \& Strong, D. (1978). The timing of marriage in the transition to adulthood: Continuity and change, 1860-1975. American Journal of Sociology, 84, 120-150.
Nolen-Hoeksema, S., Parker, L., \& Larson, J. (1994). Ruminative coping with depressed mood following loss. Journal of Personality and Social Psychology, 67, 92-104.

Radloff, L. (1977). The CES-D Scale: A self-report depression scale for research in the general population. Applied Psychological Measurement, $1,385-401$.

Rothbaum, F., Weisz, J. R., \& Snyder, S. S. (1982). Changing the world and changing the self: A two-process model of perceived control. Journal of Personality and Social Psychology, 42, 5-37.

Schulz, R., \& Heckhausen, J. (1996). A life-span model of successful aging. American Psychologist, 51, 702-714.

Schulz, R., Wrosch, C., Yee, J. L., Heckhausen, J., \& Whitmer, R. (1998, September). Avoiding depression in late life. Using selective and compensatory control processes to manage physical illness and disability. Paper presented at the XVth Biennial Meeting of the International Society for the Study of Behavioural Development, Bern, Switzerland.

Settersten, R. A., \& Hagestad, G. O. (1996). What's the latest? Cultural age deadlines for family transitions. The Gerontologist, 36, 178-188.

Warr, P., Barter, J., \& Brownbridge, G. (1983). On the independence of positive and negative affect. Journal of Personality and Social Psychol ogy, 44, 644-651.

Watson, D., Clark, L. A., \& Tellegen, A. (1988). Development and validation of brief measures of positive and negative affect: The PANAS scales. Journal of Personality and Social Psychology, 54, 1063-1070.

Wrosch, C. (1999). Entwicklungsfristen im Partnerschaftsbereich: Berzugsrahmen für Prozesse der Aktivierung und Deaktivierung von Entwicklungszielen [Developmental deadlines in the partnership domain: Reference frame for activating and deactivating developmental goals]. Münster, Germany: Waxmann.

Wrosch, C., \& Heckhausen, J. (1999). Control processes before and after passing a developmental deadline: Activation and deactivation of intimate relationship goals. Journal of Personality and Social Psychology, $77,415-427$.

Wrosch, C., \& Heckhausen, J. (in press). Being on-time or off-time: Developmental deadlines for regulating one's own development. In A. N. Perret-Clermont, J. M. Barrelet, A. Flammer, D. Miéville, J. F. Perret, \& W. Perrig (Eds.), Thinking time: A multidisciplinary perspective. Göttingen, Germany: Hogrefe \& Huber.

Wrosch, C., Schulz, R., \& Heckhausen, J. (2000). Health engagement control strategies and depression in the elderly. Manuscript submitted for publication.

Received December 30, 1999

Revision received July 25, 2000 Accepted October 19, 2000 